\title{
On diffeomorphisms over nonorientable surfaces standardly embedded in the 4-sphere
}

\author{
Susumu Hirose
}

\begin{abstract}
For a nonorientable closed surface standardly embedded in the 4-sphere, a diffeomorphism over this surface is extendable if and only if this diffeomorphism preserves the Guillou-Marin quadratic form of this embedded surface.
\end{abstract}

57Q45; 20F38, 57N05

\section{Introduction}

Let $S$ be a closed surface and $e$ be a smooth embedding of $S$ into $S^{4}$. A diffeomorphism $\phi$ over $S$ is $e$-extendable if there is an orientation-preserving diffeomorphism $\Phi$ of $S^{4}$ such that $\left.\Phi\right|_{S}=\phi$. The natural problem to ask is:

Find a necessary and sufficient condition for a diffeomorphism over $S$ to be e-extendable.

For some special embeddings of closed surfaces in 4-manifolds, we have answers to this problem (for example, by Montesinos [11], the author [4] and the author and Yasuhara [6]). An embedding $e$ of the orientable surface $\Sigma_{g}$ into $S^{4}$ is called standard if $e\left(\Sigma_{g}\right)$ is the boundary of 3-dimensional handlebody embedded in $S^{4}$. Montesinos [11] and the author [4] showed:

Theorem 1.1 ([11] for $g=1$; [4] for $g \geq 2$ ) Let $\Sigma_{g}$ be standardly embedded in $S^{4}$. An orientation-preserving diffeomorphism $\phi$ over the $\Sigma_{g}$ is extendable to $S^{4}$ if and only if $\phi$ preserves the Rokhlin quadratic form of the $\Sigma_{g}$ standardly embedded in $S^{4}$.

In this paper, we consider the same kind of problem for nonorientable surfaces embedded in $S^{4}$. Let $N_{g}$ be a connected nonorientable surface constructed from $g$ projective planes by connected sum. We call $N_{g}$ the closed nonorientable surface of genus $g$. Let $S^{3} \times[-1,1]$ be a closed tubular neighborhood of the equator $S^{3}$ in $S^{4}$. Then $S^{4}-S^{3} \times(-1,1)$ consists of two 4 -balls. An embedding os: $N_{g} \hookrightarrow S^{4}$ is $o$-standard if $o s\left(N_{g}\right) \subset S^{3} \times[-1,1]$ and as shown in Figure 1. On the level $t=0$ in this motion 


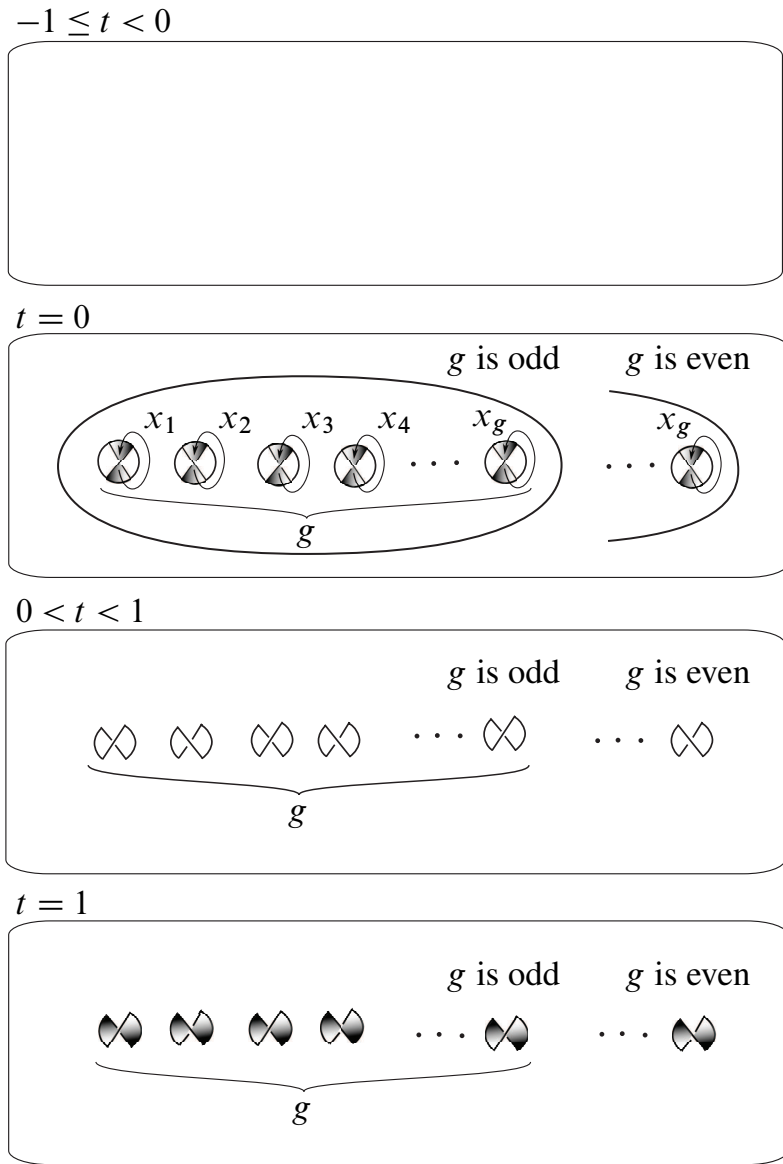

Figure 1. The motion picture of the $o$-standard embedding of $N_{g}$ into $S^{4}$
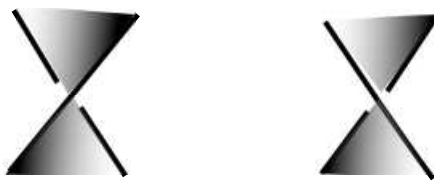

Figure 2. Bands attached on the level $t=0$ in Figure 1

picture, by the numbering of the band from the left to the right, the $(2 i-1)-$ st band is as shown in the left of Figure 2, and $2 i-$ th band is as shown in the right of Figure 2. The main result of this paper is:

Theorem 1.2 The diffeomorphism $\phi$ over $N_{g}$ is os-extendable if and only if $\phi$ preserves the Guillou-Marin quadratic form of the $N_{g}$ o-standardly embedded in $S^{4}$. 


\section{Guillou-Marin quadratic form}

For a smooth embedding $e$ of the closed nonorientable surface $N_{g}$ of genus $g$ into $S^{4}$, Guillou and Marin [3] (see also Matsumoto [10]) defined a quadratic form $q_{e}: H_{1}\left(N_{g} ; \mathbb{Z}_{2}\right) \rightarrow \mathbb{Z}_{4}$ as follows: Let $C$ be an immersed circle on $N_{g}$, and $D$ be a connected orientable surface immersed in $S^{4}$ such that $\partial D=C$, and $D$ is not tangent to $N_{g}$. Let $v_{D}$ be the normal bundle of $D$, then $\left.v_{D}\right|_{C}$ is a solid torus with a trivialization induced from any trivialization of $v_{D}$. Let $N_{N_{g}}(C)$ be the tubular neighborhood of $C$ in $N_{g}$, then $N_{N_{g}}(C)$ is a twisted annulus or a Möbius band in $\left.v_{D}\right|_{C}$. We denote by $n(D)$ the number of right-hand half-twists of $N_{N_{g}}(C)$ with respect to the trivialization of $\left.v_{D}\right|_{C}$. Let $D \cdot N_{g}$ be the mod-2 intersection number between the interior of $D$ and $N_{g}, \operatorname{Self}(C)$ be the mod-2 double points number of $C$, and $2 \times$ be an injection $\mathbb{Z}_{2} \rightarrow \mathbb{Z}_{4}$ defined by $2 \times[n]_{2}=[2 n]_{4}$. Then the number $n(D)+2 \times D \cdot N_{g}+2 \times \operatorname{Self}(C)(\bmod 4)$ depend only on the mod-2 homology class $[C]$ of $C$. Hence, we define

$$
q_{e}([C])=n(D)+2 \times D \cdot N_{g}+2 \times \operatorname{Self}(C)(\bmod 4) .
$$

This map $q_{e}$ is called Guillou-Marin quadratic form, since $q_{e}$ satisfies

$$
q_{e}(x+y)=q_{e}(x)+q_{e}(y)+2 \times(x \cdot y)_{2},
$$

where $(x \cdot y)_{2}$ is the mod-2 intersection number between $x$ and $y$. Let $\left\{x_{1}, \ldots, x_{g}\right\}$ be the basis of $H_{1}\left(N_{g} ; \mathbb{Z}_{2}\right)$ shown in Figure 1 . For $x_{1}$, let $D$ be a disc such that $\partial D=x_{1}$ and the interior of $D$ is in $S^{2} \times(0,1]$, then $D \cdot N_{g}=0$. Since $N_{N_{g}}\left(x_{1}\right)$ is a Möbius band with one right-hand half-twist, $q_{o s}\left(x_{1}\right)=+1$. By the same way as above, we see that $q_{o s}\left(x_{2 i-1}\right)=+1, q_{o s}\left(x_{2 i}\right)=-1$. This quadratic form $q_{e}$ is a nonorientable analogy of Rokhlin quadratic form.

A diffeomorphism $\phi$ over $N_{g}$ is $e$-extendable if there is an orientation-preserving diffeomorphism $\Phi$ of $S^{4}$ such that the following diagram is commutative:

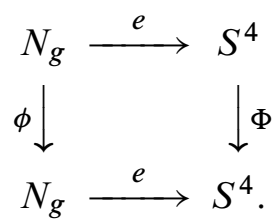

If the diffeomorphisms $\phi_{1}$ over $N_{g}$ is $e$-extendable, and $\phi_{1}$ is isotopic to $\phi_{2}$, then $\phi_{2}$ is $e$-extendable. Therefore, $e$-extendability is a property about isotopy classes of diffeomorphisms over $N_{g}$. The group $\mathcal{M}\left(N_{g}\right)$ of isotopy classes of all diffeomorphisms over $N_{g}$ is called the mapping class group of $N_{g}$. An element $\phi$ of $\mathcal{M}\left(N_{g}\right)$ is $e-$ extendable if there is an $e$-extendable representative of $\phi$. By the definition of $q_{e}$, we 
can see that if $\phi \in \mathcal{M}\left(N_{g}\right)$ is $e$-extendable then $\phi$ preserves $q_{e}$, ie $q_{e}\left(\phi_{*}(x)\right)=q_{e}(x)$ for every $x \in H_{1}\left(N_{g} ; \mathbb{Z}_{2}\right)$. What we would like to know is whether $\phi \in \mathcal{M}\left(N_{g}\right)$ is $e$-extendable when $\phi$ preserves $q_{e}$. The answer to this problem would be depend on the embedding $e$. In this paper, we consider the case where $e$ is the $o$-standard embedding.

\section{Generators for $\mathcal{M}\left(N_{g}\right)$}

A simple closed curve $c$ on $N_{g}$ is an A-circle (resp. an M-circle), if the tubular neighborhood of $c$ is an annulus (resp. a Möbius band). We denote by $t_{c}$ the Dehn twist about an A-circle $c$ on $N_{g}$. In each figure, we indicate the direction of a Dehn twist by an arrow. Lickorish [8; 9] showed that Dehn twists and $Y$-homeomorphisms generate $\mathcal{M}\left(N_{g}\right)$. We review the definition of $Y$-homeomorphism. Let $m$ be an M-circle and $a$ be an oriented A-circle in $N_{g}$ such that $m$ and $a$ transversely intersect in one point. Let $K \subset N_{g}$ be a regular neighborhood of $m \cup a$, which is a union of the tubular neighborhoods of $m$ and $a$ and then is homeomorphic to the Klein bottle with a hole. Let $M$ be a regular neighborhood of $m$. We denote by $Y_{m, a}$ a homeomorphism over $N_{g}$ which is described as the result of pushing $M$ once along $a$ keeping the boundary of $K$ fixed (see Figure 3). We call $Y_{m, a}$ a $Y$-homeomorphism.

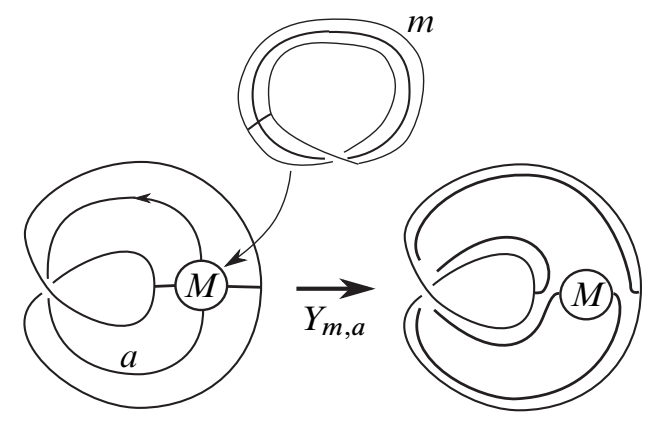

Figure 3. $M$ with circle indicates a place where to attach a Möbius band

Szepietowski [13] showed an interesting results on the proper subgroup of $\mathcal{M}\left(N_{g}\right)$ generated by all $Y$-homeomorphisms.

Theorem 3.1 [13] $\Gamma_{2}\left(N_{g}\right)=\left\{\phi \in \mathcal{M}\left(N_{g}\right) \mid \phi_{*}: H_{1}\left(N_{g} ; \mathbb{Z}_{2}\right) \rightarrow H_{1}\left(N_{g} ; \mathbb{Z}_{2}\right)=\mathrm{id}\right\}$ is generated by $Y$-homeomorphisms.

Chillingworth [2] showed that $\mathcal{M}\left(N_{g}\right)$ is finitely generated. In this paper, we use the system of generators of $\mathcal{M}\left(N_{g}\right)$ listed by Szepietowski [13]: 
Theorem 3.2 [13, Theorem 3.3] Let $a_{1}, \ldots, a_{g-1}, b_{j}(1 \leq j \leq g / 2)$ and $m_{g-1}$ be circles shown in Figure 4. Then $t_{a_{1}}, \ldots, t_{a_{g-1}}, t_{b_{j}}(1 \leq j \leq g / 2), Y_{m_{g-1}, a_{g-1}}$ generate $\mathcal{M}\left(N_{g}\right)$.

$g$ is even

$g$ is odd
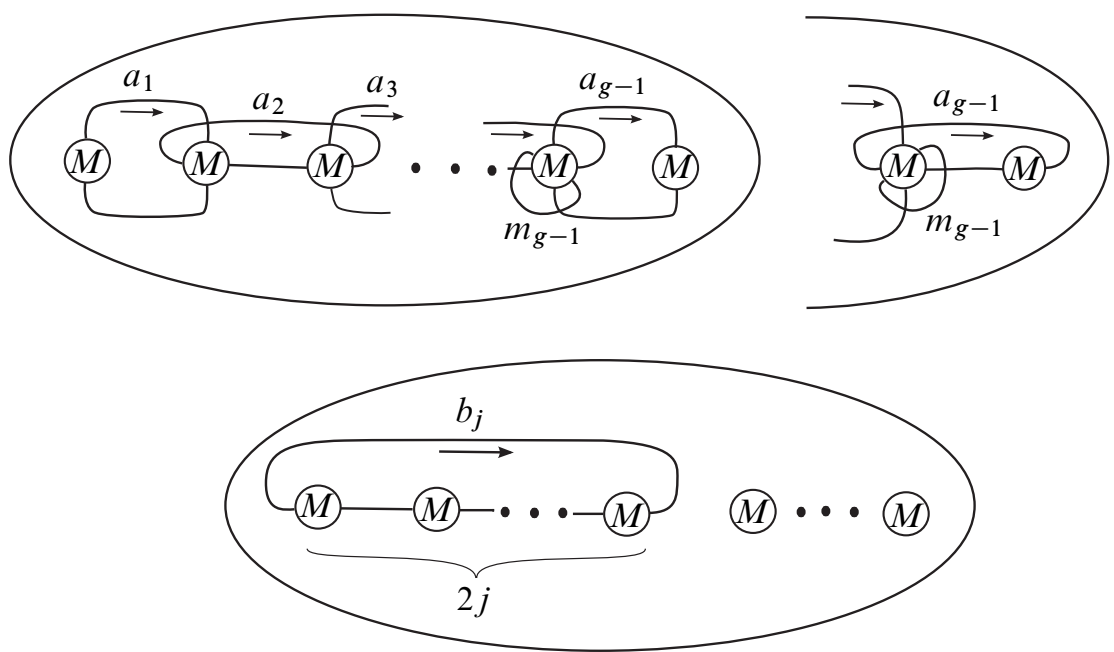

Figure 4. Generators for $\mathcal{M}\left(N_{g}\right)$

Remark 3.3 If $g=1$, then $\mathcal{M}\left(N_{1}\right)$ is trivial, hence Theorem 1.2 is valid. From here to the end of this paper, we assume $g \geq 2$.

The $i$-th band is the Möbius band on which the circle $x_{i}$ in Figure 1 goes across. Let $c_{i}(i=1, \ldots, g-3)$ be a simple closed curve shown in the top of Figure 5. Let $i, j=1, \ldots, g$ such that $i \neq j$. When $i<j$ (resp. $i>j$ ), we define $Y_{i, j}=Y_{m, a}$, where $m$ and $a$ are as shown in the bottom left (resp. the bottom right) of Figure 5 . Let $\mathcal{Y} \mathcal{S}_{g}$ be the subgroup of $\mathcal{M}\left(N_{g}\right)$ generated by all $Y_{i, j}$.

Lemma $3.4 \mathcal{Y} \mathcal{S}_{g}$ and $t_{a_{1}}, \ldots, t_{a_{g-1}}, t_{c_{1}}, \ldots, t_{c_{g-3}}$ generate $\mathcal{M}\left(N_{g}\right)$.

Proof It suffices to show that $t_{b_{j}}$ is a product of $t_{a_{1}}, \ldots, t_{a_{g-1}}, t_{c_{1}}, \ldots, t_{c_{g-3}}$. When $j=2, t_{b_{2}}=t_{c_{1}}$. When $j \geq 3$, by the lantern relation (discovered by Dehn and rediscovered by Johnson [7]), $t_{e_{1}} t_{e_{2}} t_{e_{3}}=t_{p} t_{a_{2 j-3}} t_{a_{2 j-1}} t_{b_{j}}$, where $p, e_{1}, e_{2}$ and $e_{3}$ are circles shown in Figure 6 , hence $t_{b_{j}}=\left(t_{p} t_{a_{2 j-3}} t_{a_{2 j-1}}\right)^{-1} t_{e_{1}} t_{e_{2}} t_{e_{3}}$. Since $e_{1}=$ $t_{a_{2 j-2}} t_{a_{2 j-3}} t_{a_{2 j-1}} t_{a_{2 j-2}}\left(e_{3}\right)$, we see $t_{e_{3}}$ is the product of $\left(t_{a_{2 j-2}} t_{a_{2 j-3}} t_{a_{2 j-1}} t_{a_{2 j-2}}\right)^{-1}$ and $t_{e_{1}} t_{a_{2 j-2}} t_{a_{2 j-3}} t_{a_{2 j-1}} t_{a_{2 j-2}}$. If $j=3$, then $e_{1}=c_{1}, e_{2}=c_{3}$ and $p=a_{1}$. Therefore, $t_{b_{3}}$ is a product of $t_{a_{1}}, \ldots, t_{a_{g-1}}, t_{c_{1}}, \ldots, t_{c_{g-3}}$. If $j \geq 4$, then $e_{1}=b_{j-1}, e_{2}=c_{2 j-3}$, and $p=b_{j-2}$. By the induction on $j$, we see that $t_{b_{j}}$ is a product of $t_{a_{1}}, \ldots, t_{a_{g-1}}$, $t_{c_{1}}, \ldots, t_{c_{g-3}}$. 


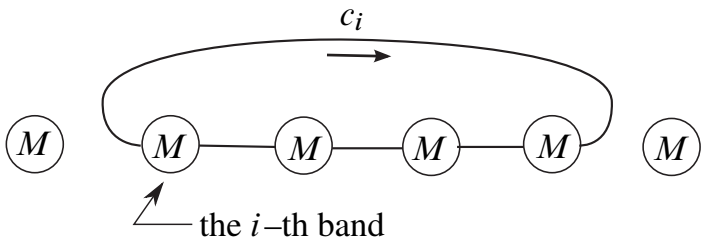

the $j$-th band the $j$-th band

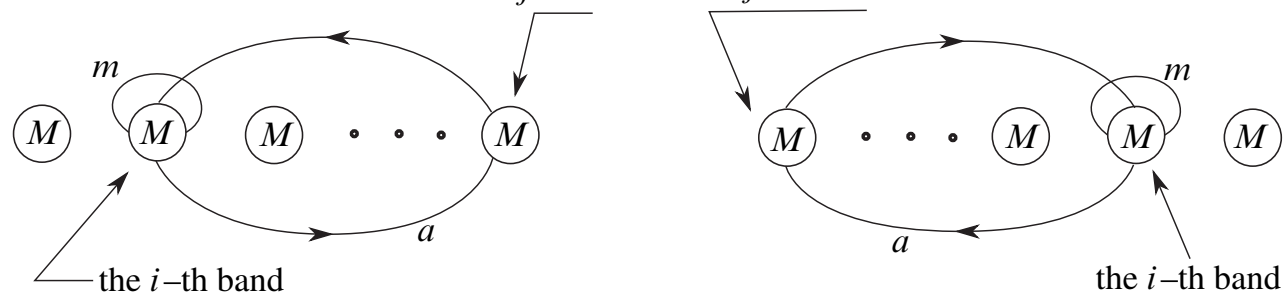

Figure 5. The circle $c_{i}$ and the $Y$-homeomorphism $Y_{i, j}$
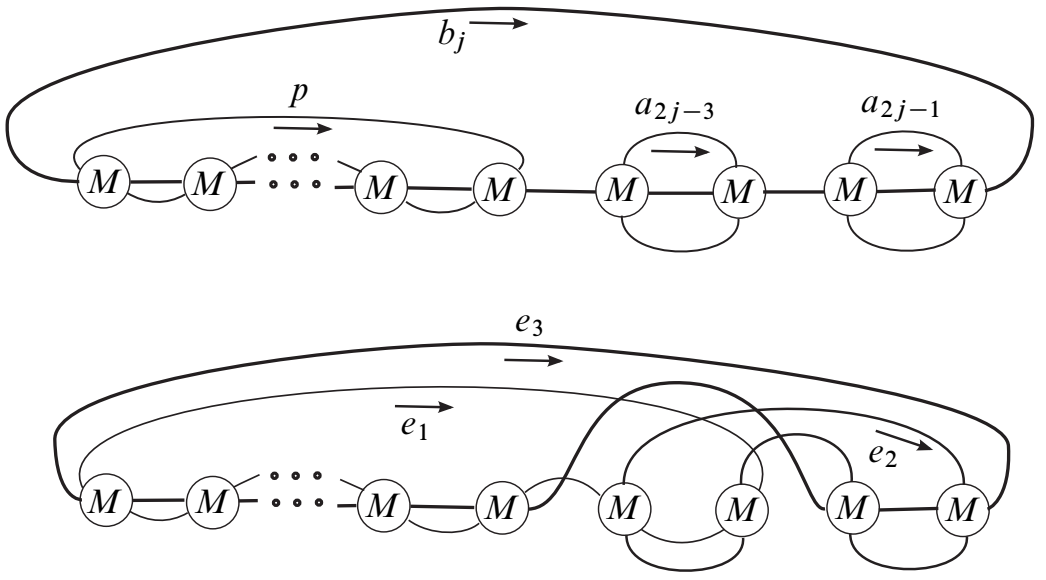

Figure 6. When $j=3$, we drop the second and third $M$ with circles.

Remark 3.5 If $g=2,3$, then this lemma reads $\mathcal{Y} \mathcal{S}_{g}$ and $t_{a_{1}}, \ldots, t_{a_{g-1}}$ generate $\mathcal{M}\left(N_{g}\right)$.

\section{Generators for subgroup of $\mathcal{M}\left(N_{g}\right)$ preserving qos}

In this section, we find a finite system of generators for

$$
\mathcal{N}_{g}\left(q_{o s}\right)=\left\{\phi \in \mathcal{M}\left(N_{g}\right) \mid q_{o s}\left(\phi_{*}(x)\right)=q_{o s}(x) \text { for every } x \in H_{1}\left(N_{g} ; \mathbb{Z}_{2}\right)\right\}
$$

and prove the main theorem (Theorem 1.2) of this paper. 
We introduce a group

$\mathcal{O}_{g}\left(q_{o s}\right)=\left\{A \in \operatorname{Aut}\left(H_{1}\left(N_{g} ; \mathbb{Z}_{2}\right)\right) \mid q_{o s}(A(x))=q_{o s}(x)\right.$ for every $\left.x \in H_{1}\left(N_{g} ; \mathbb{Z}_{2}\right)\right\}$.

Then we have a natural short exact sequence

$$
1 \rightarrow \Gamma_{2}\left(N_{g}\right) \rightarrow \mathcal{N}_{g}\left(q_{o s}\right) \rightarrow \mathcal{O}_{g}\left(q_{o s}\right) \rightarrow 1
$$

Since $\Gamma_{2}\left(N_{g}\right)$ is a finite index subgroup of $\mathcal{M}\left(N_{g}\right)$ and $\mathcal{O}_{g}\left(q_{o s}\right)$ is a finite group, there exists a finite system of generators for $\mathcal{N}_{g}\left(q_{o s}\right)$. We find a system of generators explicitly.

Theorem 4.1 The group $\mathcal{N}_{g}\left(q_{o s}\right)$ is generated by $\mathcal{Y} \mathcal{S}_{g}, t_{a_{1}}^{2}, \ldots, t_{a_{g-1}}^{2}, t_{c_{1}}^{2}, \ldots, t_{c_{g-3}}^{2}$, $t_{d_{1}}, \ldots, t_{d_{g-2}}$, and $t_{a_{1}} t_{a_{3}} t_{c_{1}}, \ldots, t_{a_{g-3}} t_{a_{g-1}} t_{c_{g-3}}$, where $d_{i}$ is illustrated in Figure 7 .
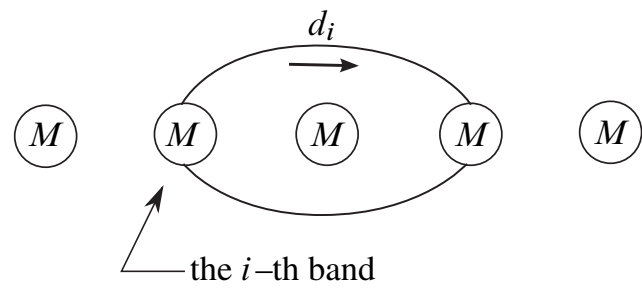

Figure 7. The circle $d_{i}$

Remark 4.2 If $g=2$, this theorem reads $\mathcal{N}_{2}\left(q_{o s}\right)$ is generated by $\mathcal{Y} \mathcal{S}_{2}$ and $t_{a_{1}}^{2}=\mathrm{id}_{N_{2}}$. If $g=3$, this theorem reads $\mathcal{N}_{3}\left(q_{o s}\right)$ is generated by $\mathcal{Y} \mathcal{S}_{3}, t_{a_{1}}^{2}, t_{a_{2}}^{2}$ and $t_{d_{1}}$.

Proof of Theorem 1.2 By the definition of $q_{o s}$, if a diffeomorphism $\phi$ over $N_{g}$ is os-extendable then $\phi$ preserves $q_{o s}$.

Conversely, we assume that $\phi$ preserves $q_{o s}$. Then $\phi$ is an element of $\mathcal{N}_{g}\left(q_{o s}\right)$. Therefore, if each generator of $\mathcal{N}_{g}\left(q_{o s}\right)$ is $o s$-extendable then $\phi$ is $o s$-extendable.

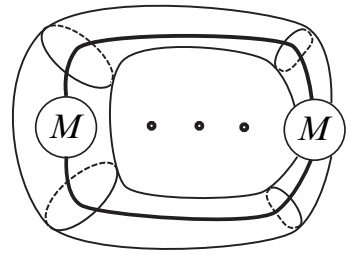

Figure 8. Sliding the left Möbius band along this tube is an extension of $Y_{i, j}$.

Since a sliding of a Möbius band along the tube illustrated in Figure 8 is an extension of $Y_{i, j}, Y_{i, j}$ is $o s$-extendable, hence every element of $\mathcal{Y} \mathcal{S}_{g}$ is $o s$-extendable. Since 
the regular neighborhoods of $a_{i}$ and $c_{i}$ are annuli trivially embedded in the equator $S^{3}$ of $S^{4}, t_{a_{i}}^{2}, t_{c_{i}}^{2}$ are $o s$-extendable by the same argument as in the introduction of [5]. Since the regular neighborhoods of $d_{i}$ is a Hopf band embedded in the equator $S^{3}$ of $S^{4}, t_{d_{i}}$ are $o s$-extendable by the same argument as the proof of [5, Proposition 2.1]. Finally, by using the same argument as showing the extendability of " $C_{1} C_{3} C_{5}$ " in the proof of [4, Lemma 2.2], we show that $t_{a_{i}} t_{a_{i+2}} t_{c_{i}}$ is $o s$-extendable.

As shown in the above proof of Theorem 1.2, any elements of $\mathcal{Y} \mathcal{S}_{g}, t_{a_{1}}^{2}, \ldots, t_{a_{g-1}}^{2}$, $t_{c_{1}}^{2}, \ldots, t_{c_{g-3}}^{2}, t_{d_{1}}, \ldots, t_{d_{g-2}}$, and $t_{a_{1}} t_{a_{3}} t_{c_{1}}, \ldots, t_{a_{g-3}} t_{a_{g-1}} t_{c_{g-3}}$ are os-extendable, hence these elements preserve $q_{o s}$. Therefore, in order to prove Theorem 4.1, we should see that every element of $\mathcal{N}_{g}\left(q_{o s}\right)$ is a product of these elements.

\subsection{Short-leg $Y$-homeomorphisms}

For a $Y$-homeomorphism $Y_{m, a}$, we call $m$ the leg of $Y_{m, a}$ and $a$ the $a r m$ of $Y_{m, a}$. A $Y$-homeomorphism is called a short-leg $Y$-homeomorphism, if its leg is one of $x_{1}, \ldots, x_{g}$ illustrated in Figure 1.

Lemma 4.3 Every short-leg $Y$-homeomorphism is an element of $\mathcal{Y} \mathcal{S}_{g}$.

Proof We review the crosscap pushing map defined in [14]. Fix $p_{0} \in N_{g-1}$ and define $\mathcal{M}\left(N_{g-1}, p_{0}\right)$ be the group of isotopy classes of diffeomorphisms over $N_{g-1}$ preserving $p_{0}$. Let $U$ be a 2-disk embedded in $N_{g-1}$ such that the center of $U$ is $p_{0}$. We parametrize $U$ by $\left\{(x, y) \mid x^{2}+y^{2} \leq 1\right\}$ such that $(0,0)=p_{0}$. Under this parametrization, we define an orientation reversing diffeomorphism $r: U \rightarrow U$ by $r(x, y)=(x,-y)$. We define a homomorphism $j$ from $\pi_{1}\left(N_{g-1}, p_{0}\right)$ to $\mathcal{M}\left(N_{g-1}, p_{0}\right)$ such that, for a loop $\gamma$ in $N_{g}$ based at $x_{0}$ and an element $[\gamma] \in \pi_{1}\left(N_{g-1}, x_{0}\right), j([\gamma])$ is a diffeomorphism over $N_{g}$ obtained as the effect of pushing $p_{0}$ once along $\gamma$. This homomorphism $j$ is a homomorphism in a nonorientable analogy of the Birman exact sequence [1]. We define a homomorphism $\varphi$ from $\mathcal{M}\left(N_{g-1}, p_{0}\right)$ to $\mathcal{M}\left(N_{g}\right)$ as follows. We represent $h \in \mathcal{M}\left(N_{g-1}, p_{0}\right)$ by a diffeomorphism $h$ over $N_{g}$ such that $h(U)=U$ and $\left.h\right|_{U}=\operatorname{id}_{U}$ or $\left.h\right|_{U}=r$. We construct $N_{g}$ from $N_{g-1}-\operatorname{int} U$ by attaching a Möbius band along $\partial U$. Here we assume that this Möbius band is the $i$-th band on $N_{g}$. We extend $\left.h\right|_{N_{g-1}-\text { int } U}$ to a diffeomorphism $\varphi(h)$ over $N_{g}$ naturally. The homomorphism $\psi=\varphi \circ j$ is called a crosscap pushing map.

Every short-leg $Y$-homeomorphism $Y_{x_{i}, a}$ is in $\psi\left(\pi_{1}\left(N_{g-1}, p_{0}\right)\right), \pi_{1}\left(N_{g-1}, p_{0}\right)$ is generated by the loops $l_{i, j}$ 's indicated in Figure 9 , and $\psi\left(l_{i, j}\right)=Y_{i, j}$, hence $Y_{x_{i}, a}$ is a product of $Y_{i, j}$ 's. 


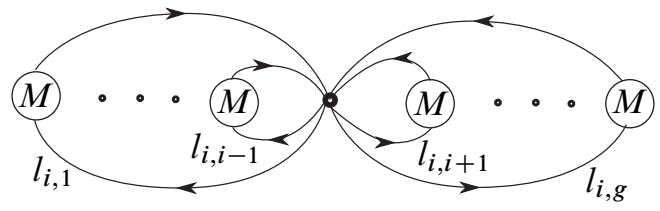

Figure 9. The generators for $\pi_{1}\left(N_{g}, p_{0}\right)$

Let $G_{g}$ be the subgroup of $\mathcal{M}\left(N_{g}\right)$ generated by $\mathcal{Y} \mathcal{S}_{g}, t_{a_{1}}^{2}, \ldots, t_{a_{g-1}}^{2}, t_{c_{1}}^{2}, \ldots, t_{c_{g-3}}^{2}$, $t_{d_{1}}, \ldots, t_{d_{g-2}}, t_{a_{1}} t_{a_{3}} t_{c_{1}}, \ldots, t_{a_{g-3}} t_{a_{g-1}} t_{c_{g-3}}$. We have already shown that $G_{g} \subset$ $\mathcal{N}_{g}\left(q_{o s}\right)$, therefore, what we should show is $\mathcal{N}_{g}\left(q_{o s}\right) \subset G_{g}$. Two $Y$-homeomorphisms $Y_{1}$ and $Y_{2}$ are $G_{g}$-equivalent if there is an element $\phi$ of $G_{g}$ such that $\phi Y_{1} \phi^{-1}=Y_{2}$. We remark that if $Y_{1}=Y_{m, a}$ and $Y_{2}=\phi Y_{1} \phi^{-1}$ then $Y_{2}=Y_{\phi(m), \phi(a)}$. We will show:

Lemma 4.4 Every $Y$-homeomorphism is a product of $Y$-homeomorphisms which are $G_{g}$-equivalent to short-leg $Y$-homeomorphisms.

By Lemma 4.3 and Lemma 4.4, we see that every $Y$-homeomorphism is an element of $G_{g}$. Therefore, by Theorem 3.1, we conclude:

Corollary $4.5 \Gamma_{2}\left(N_{g}\right) \subset G_{g}$.

Remark 4.6 While the author was writing this paper, Błażej Szepietowski informed the author that he found a finite system of generators for $\Gamma_{2}\left(N_{g}\right)$. In the next subsection, we introduce his system of generators and prove Lemma 4.4 by using his result. In this subsection, we show Lemma 4.4 by our original proof.

As shown in Figure 10, we use the symbol $\oplus$ (resp. $\ominus$ ) to indicate the place where the $g$ is odd $g$ is even

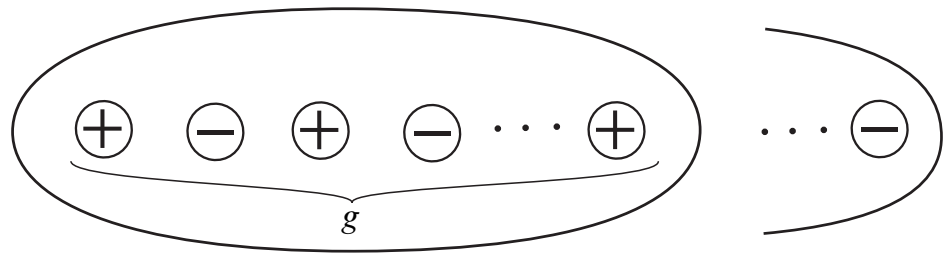

Figure 10. Diagram indicating $o-$ standard $N_{g}$ in $S^{4}$

Möbius band are attached such that $q_{o s}\left(x_{i}\right)=+1$ (resp. $\left.q_{o s}\left(x_{i}\right)=-1\right)$ for the circle $x_{i}$ indicated in Figure 1. We denote an element $x=\sum_{i=1}^{g} \epsilon_{i} x_{i} \in H_{1}\left(N_{g} ; \mathbb{Z}_{2}\right)$, where $\epsilon_{i}=0$ or 1 , by a sequence of symbols $+,-, \oplus, \ominus$ of length $g$ with [, ] which are 
settled by the rule: if $\epsilon_{2 i-1}=0$ then the (2i-1)-st symbol is + , if $\epsilon_{2 i-1}=1$ then the $(2 i-1)$-st symbol is $\oplus$, if $\epsilon_{2 i}=0$ then the $2 i$-th symbol is - , and if $\epsilon_{2 i}=1$ then the $2 i$-th symbol is $\ominus$. For example, when $g=7$, we denote an element $x_{2}+x_{3}+x_{6}+x_{7}$ by $[+\ominus \oplus-+\ominus \oplus]$. This sequence is called the $r$-sequence associated to $x$. For the r-sequence associated to $x$, we settle a simple closed curve on $N_{g}$ by the following rule. For the symbols in this sequence, we put arcs on $N_{g}$ indicated in the bottom of

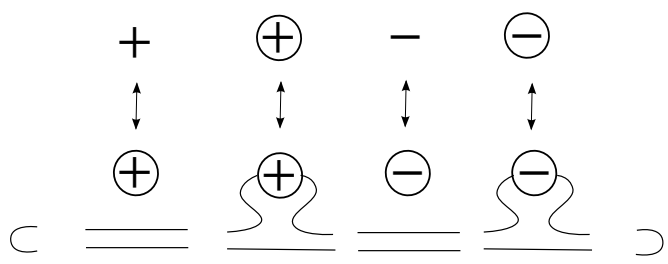

Figure 11. Parts of $\mathrm{r}-$ circles

Figure 11, glue them along the boundaries, and cap by the arc indicated on the left of Figure 11 from the left and by the arc indicated on the right of Figure 11 from the right. We call this circle the $r$-circle associated to $x$ and denote by $R(x)$. For an element $x=\sum_{i=1}^{g} \epsilon_{i} x_{i} \in H_{1}\left(N_{g} ; \mathbb{Z}_{2}\right)$, where $\epsilon_{i}=0$ or 1 , we define $\operatorname{supp}(x)=\left\{x_{i} \mid \epsilon_{i}=1\right\}$.

Two simple closed curves $c_{1}$ and $c_{2}$ on $N_{g}$ are $G_{g}$-equivalent $\left(c_{1} \sim_{G_{g}} c_{2}\right)$ if there is an element $\phi$ of $G_{g}$ such that $\phi\left(c_{1}\right)=c_{2}$.

Lemma 4.7 If $g=1$, then every $r$-circle is $G_{g}$-equivalent to $R([+])$ or $R([\oplus])$. If $g=2$, then every $r$-circle is $G_{g}$-equivalent to $R([+-]), R([\oplus-]), R([+\ominus])$ or $R([\oplus \ominus])$. If $g \geq 3$ is odd, then every $r$-circle is $G_{g}$-equivalent to $R([+-+-\cdots+])$, $R([\oplus-+-\cdots+]), R([+\ominus+-\cdots+]), R([\oplus \ominus+-\cdots+]), R([\oplus-\oplus-\cdots+])$ or $R([\oplus \ominus \oplus \ominus \cdots \oplus])$. If $g \geq 4$ is even, then every r-circle is $G_{g}$-equivalent to $R([+-+-\cdots-]), R([\oplus-+-\cdots-]), R([+\ominus+-\cdots-]), R([\oplus \ominus+-\cdots-])$, $R([\oplus-\oplus-\cdots-])$ or $R([\oplus \ominus \oplus \ominus \cdots \ominus])$.

Proof If $g=1$ or 2 , then the conclusion is trivial.

If $g \geq 3$, then

$$
\begin{aligned}
& R([\cdots-+\ominus \cdots]) \sim_{G_{g}} R([\cdots \ominus+-\cdots]), \\
& R([\cdots+-\oplus \cdots]) \sim_{G_{g}} R([\cdots \oplus-+\cdots]), \\
& R([\cdots-\oplus \ominus \cdots]) \sim_{G_{g}} R([\cdots \ominus \oplus-\cdots]), \\
& R([\cdots+\ominus \oplus \cdots]) \sim_{G_{g}} R([\cdots \oplus \ominus+\cdots]),
\end{aligned}
$$


where the leftmost symbols are the $i$-th symbol, since

$$
\begin{aligned}
Y_{i+2, i} Y_{i+1, i} t_{d_{i}} R([\cdots-+\ominus \cdots]) & =R([\cdots \ominus+-\cdots]), \\
Y_{i+2, i} Y_{i+1, i} t_{d_{i}} R([\cdots+-\oplus \cdots]) & =R([\cdots \oplus-+\cdots]), \\
Y_{i+2, i+1} Y_{i+1, i+2} t_{d_{i}} R([\cdots-\oplus \ominus \cdots]) & =R([\cdots \ominus \oplus-\cdots]), \\
Y_{i+2, i+1} Y_{i+1, i+2} t_{d_{i}} R([\cdots+\ominus \oplus \cdots]) & =R([\cdots \oplus \ominus+\cdots]) .
\end{aligned}
$$

Therefore, when $g=3$, for two cases $R([+-\oplus])$ and $R([+\ominus \oplus])$ which are not listed in the statement, we see $R([+-\oplus]) \sim_{G_{g}} R([\oplus-+])$ and $R([+\ominus \oplus]) \sim_{G_{g}} R([\oplus \ominus+])$.

If $g \geq 4$, then

$$
\begin{aligned}
& R([\cdots-\oplus \ominus \oplus \cdots]) \sim_{G_{g}} R([\cdots-\oplus-+\cdots]), \\
& R([\cdots+\ominus \oplus \ominus \cdots]) \sim_{G_{g}} R([\cdots+\ominus+-\cdots]), \\
& R([\cdots \ominus \oplus \ominus+\cdots]) \sim_{G_{g}} R([\cdots-+\ominus+\cdots]), \\
& R([\cdots \oplus \ominus \oplus-\cdots]) \sim_{G_{g}} R([\cdots+-\oplus-\cdots]), \\
& R([\cdots-\oplus-\oplus \cdots]) \sim_{G_{g}} R([\cdots \ominus+\ominus+\cdots]), \\
& R([\cdots+\ominus+\ominus \cdots]) \sim_{G_{g}} R([\cdots \oplus-\oplus-\cdots]),
\end{aligned}
$$

where the leftmost symbols are the $i$-th symbol, since

$$
\begin{aligned}
Y_{i+3, i+1} Y_{i+2, i+1} t_{a_{i}}^{-2}\left(t_{a_{i}} t_{a_{i+2}} t_{c_{i}}\right) R([\cdots-\oplus \ominus \oplus \cdots]) & =R([\cdots-\oplus-+\cdots]), \\
Y_{i+3, i+1} Y_{i+2, i+1} t_{a_{i}}^{-2}\left(t_{a_{i}} t_{a_{i+2}} t_{c_{i}}\right) R([\cdots+\ominus \oplus \ominus \cdots]) & =R([\cdots+\ominus+-\cdots]), \\
Y_{i, i+2} Y_{i+1, i+2} t_{a_{i+2}}^{2}\left(t_{a_{i}} t_{a_{i+2}} t_{c_{i}}\right)^{-1} R([\cdots \ominus \oplus \ominus+\cdots]) & =R([\cdots-+\ominus+\cdots]), \\
Y_{i, i+2} Y_{i+1, i+2} t_{a_{i+2}}^{2}\left(t_{a_{i}} t_{a_{i+2}} t_{c_{i}}\right)^{-1} R([\cdots \oplus \ominus \oplus-\cdots]) & =R([\cdots+-\oplus-\cdots]), \\
Y_{i+1, i}\left(t_{a_{i}} t_{a_{i+2}} t_{c_{i}}\right) Y_{i+2, i+3}^{-1} R([\cdots-\oplus-\oplus \cdots]) & =R([\cdots \ominus+\ominus+\cdots]), \\
Y_{i+1, i}\left(t_{a_{i}} t_{a_{i+2}} t_{c_{i}}\right) Y_{i+2, i+3}^{-1} R([\cdots+\ominus+\ominus \cdots]) & =R([\cdots \oplus-\oplus-\cdots]) .
\end{aligned}
$$

When $g \geq 4$, we get our conclusion by the induction on $g$ and $G_{g}$-equivalences (2) and (3). When $g \geq 4$ is even, by the induction hypothesis, every r-circle of length $g-1$ is $G_{g-1}$-equivalent to $R([+-+-\cdots+]), R([\oplus-+-\cdots+]), R([+\ominus+-\cdots+])$, $R([\oplus \ominus+-\cdots+]), R([\oplus-\oplus-\cdots+])$ or $R([\oplus \ominus \oplus \ominus \cdots \oplus])$, therefore every r-circle of length $g$ is $G_{g}$ equivalent to

(i) $R([+-+-\cdots+-])$,

(ii) $R([+-+-\cdots+\ominus])$,

(iii) $R([\oplus-+-\cdots+-])$,

(iv) $R([\oplus-+-\cdots+\ominus])$, 
(v) $R([+\ominus+-\cdots+-])$,

(vi) $R([+\ominus+-\cdots+\ominus])$,

(vii) $R([\oplus \ominus+-\cdots+-])$,

(viii) $R([\oplus \ominus+-\cdots+\ominus])$,

(ix) $R([\oplus-\oplus-\cdots+-])$,

(x) $R([\oplus-\oplus-\cdots+\ominus])$,

(xi) $R([\oplus \ominus \oplus \ominus \cdots \oplus-])$,

(xii) $R([\oplus \ominus \oplus \ominus \cdots \oplus \ominus])$.

By applying $G_{g}$-equivalences (2) and (3), we see the elements (ii), (iv), (vi), (viii), (x) and (xi) are $G_{g}$-equivalent to one of other six elements. For example,

$$
\begin{aligned}
\text { (viii) }=R([\oplus \ominus+-\cdots+\ominus]) & \sim_{G_{g}} R([\oplus \ominus+\ominus \cdots+-]) \\
& \sim_{G_{g}} R([+\ominus \oplus \ominus \cdots+-]) \\
& \sim_{G_{g}} R([+\ominus+-\cdots+-])=(\mathrm{v}) .
\end{aligned}
$$

By the same method as above, we get our conclusion when $g \geq 4$ is odd.

If the complement of an M-circle $m$ is orientable, then any circle intersecting $m$ transversely in one point is an $\mathrm{M}$-circle. Therefore the leg of every $Y$-homeomorphism is an M-circle whose complement is nonorientable. Every element of $G_{g}$ preserves $q_{o s}$, the $\mathrm{r}-$ circles $R([+-\cdots \pm]), R([\oplus-\oplus-\cdots \pm])$ and $R([\oplus \ominus+\cdots \pm])$ are A-circles, and the complements of $R([\oplus \ominus \cdots \oplus])$ and $R([\oplus \ominus \cdots \ominus])$ are orientable, hence:

Corollary 4.8 If an r-circle $R(x)$ is a leg of a $Y$-homeomorphism, then $R(x)$ is $G_{g}$-equivalent to $R([\oplus-+\cdots])$ or $R([+\ominus+\cdots])$.

By investigating the action of generators for $\mathcal{M}\left(N_{g}\right)$ listed in Lemma 3.4 on legs of $Y$-homeomorphisms, we see:

Lemma 4.9 Every $Y$-homeomorphism is a product of $Y$-homeomorphisms whose legs are $r-$ circles.

Proof Since $\left\{x_{1}, \ldots, x_{g}\right\}$ are $\mathrm{r}$-circles, $Y_{i, j}$ is a $Y$-homeomorphism whose leg is an r-circle. For every $Y$-homeomorphism $Y_{m, a}$, there is an r-circle $s$ and an element $\phi \in \mathcal{M}\left(N_{g}\right)$ such that $\phi(s)=m$, that is, $Y_{m, a}=\phi Y_{s, \phi^{-1}(a)} \phi^{-1}$. Therefore, by Lemma 3.4, it suffices to show that for every r-circle $s$ there are $\phi_{i}, \phi_{i}^{\prime}, \psi_{i}, \psi_{i}^{\prime} \in \mathcal{Y} \mathcal{S}_{g}$ and $\mathrm{r}-$ circles $s_{i}, s_{i}^{\prime}, t_{i}, t_{i}^{\prime}$ such that $t_{a_{i}}(s)=\phi_{i}\left(s_{i}\right), t_{a_{i}}^{-1}(s)=\phi_{i}^{\prime}\left(s_{i}^{\prime}\right), t_{c_{i}}(s)=\psi_{i}\left(t_{i}\right)$ 
and $t_{c_{i}}^{-1}(s)=\psi_{i}^{\prime}\left(t_{i}^{\prime}\right)$. As observed in the proof of [13, Lemma 3.1], $Y_{x_{i}, a_{i}}$ preserves $a_{i}$ and exchanges the sides of $a_{i}$, hence $t_{a_{i}}=Y_{x_{i}, a_{i}} t_{a_{i}}^{-1} Y_{x_{i}, a_{i}}^{-1}$, therefore $t_{a_{i}}^{2}=t_{a_{i}} Y_{x_{i}, a_{i}} t_{a_{i}}^{-1} Y_{x_{i}, a_{i}}^{-1}=Y_{t_{a_{i}}\left(x_{i}\right), a_{i}} Y_{x_{i}, a_{i}}^{-1}=Y_{i+1, i} Y_{i, i+1}^{-1}$. By the same way as above, we see that $t_{c_{i}}=Y_{x_{i}, c_{i}} t_{c_{i}}^{-1} Y_{x_{i}, c_{i}}^{-1}$, therefore $t_{c_{i}}^{2}=t_{c_{i}} Y_{x_{i}, c_{i}} t_{c_{i}}^{-1} Y_{x_{i}, c_{i}}^{-1}=Y_{t_{c_{i}}\left(x_{i}\right), c_{i}} Y_{x_{i}, c_{i}}^{-1}$. Since $Y_{g, i+3} \cdots Y_{i+4, i+3} Y_{i, i+1} \cdots Y_{1, i+1} t_{c_{i}}\left(x_{i}\right)$ is isotopic to $R\left(x_{i+1}+x_{i+2}+x_{i+3}\right)$, $t_{c_{i}}^{2}$ is a product of $Y$-homeomorphisms whose legs are r-circles. From the above observation, it suffices to show that one of $t_{a_{i}}(s)=\phi_{i}\left(s_{i}\right), t_{a_{i}}^{-1}(s)=\phi_{i}^{\prime}\left(s_{i}^{\prime}\right)$, and one of $t_{c_{i}}(s)=\psi_{i}\left(t_{i}\right), t_{c_{i}}^{-1}(s)=\psi_{i}^{\prime}\left(t_{i}^{\prime}\right)$.

Since $a_{i}$ does not intersects $R(x)$ such that $\operatorname{supp}(x) \cap\left\{x_{i}, x_{i+1}\right\}=\varnothing$, we only consider the action of $t_{a_{i}}$ on $R(x)$ such that $\operatorname{supp}(x) \cap\left\{x_{i}, x_{i+1}\right\} \neq \varnothing$. When we consider the action of $t_{a_{i}}$ and $Y$-homeomorphisms, we do not need to take care of the sign on the Möbius bands. Hence, in symbols of $r$-sequences, we change + and - into $\times$, and $\oplus$ and $\ominus$ into $\otimes$. There are 3 cases to consider: $R([\cdots \otimes \times \cdots])$, $R([\cdots \times \otimes \cdots])$, and $R([\cdots \otimes \otimes \cdots])$, where the $i$-th and $(i+1)-$ st symbols are indicated. The third $\mathrm{r}-$ circle does not intersect $a_{i}$, hence we ignore this. By drawing figures of r-circles, we see: $Y_{i, i+1}\left(t_{a_{i}}^{-1}(R([\cdots \otimes \times \cdots]))\right)=R([\cdots \times \otimes \cdots])$ and $Y_{i+1, i}\left(t_{a_{i}}(R([\cdots \times \otimes \cdots]))\right)=R([\cdots \otimes \times \cdots])$.

By the same reasons as in the previous paragraph, it suffice to consider the action of $t_{c_{i}}$ on $R(x)$ such that $\operatorname{supp}(x) \cap\left\{x_{i}, x_{i+1}, x_{i+2}, x_{i+3}\right\} \neq \varnothing$, and, in symbols of r-sequences, we change + and - into $\times$, and $\oplus$ and $\ominus$ into $\otimes$. There are 15 cases to consider:

(1) $R([\cdots \otimes \times \times \times \cdots])$,

(2) $R([\cdots \times \otimes \times \times \cdots])$,

(3) $R([\cdots \otimes \otimes \times \times \cdots])$,

(4) $R([\cdots \times \times \otimes \times \cdots])$,

(5) $R([\cdots \otimes \times \otimes \times \cdots])$,

(6) $R([\cdots \times \otimes \otimes \times \cdots])$,

(7) $R([\cdots \otimes \otimes \otimes \times \cdots])$,

(8) $R([\cdots \times \times \times \otimes \cdots])$,

(9) $R([\cdots \otimes \times \times \otimes \cdots])$,

(10) $R([\cdots \times \otimes \times \otimes \cdots])$,

(11) $R([\cdots \otimes \otimes \times \otimes \cdots])$,

(12) $R([\cdots \times \times \otimes \otimes \cdots])$,

(13) $R([\cdots \otimes \times \otimes \otimes \cdots])$,

(14) $R([\cdots \times \otimes \otimes \otimes \cdots])$,

(15) $R([\cdots \otimes \otimes \otimes \otimes \cdots])$, 
where the $i-$ th, $(i+1)-$ st, $(i+2)-$ nd and $(i+3)-$ rd symbols are indicated. Since (3), (6), (12) and (15) do not intersect $c_{i}, t_{c_{i}}$ does not change these r-circles. By drawing figures of $\mathrm{r}$-circles (for example, Figure 12 indicates (9)), we see:

(1) $Y_{i, i+1} Y_{i+2, i+3} Y_{i+1, i+3} Y_{i+1, i+2}^{-1}\left(t_{c_{i}}^{-1}(R([\cdots \otimes \times \times \times \cdots]))\right)$ $=R([\cdots \times \otimes \otimes \otimes \cdots])$,

(2) $Y_{i+1, i} Y_{i+2, i+3}\left(t_{c_{i}}(R([\cdots \times \otimes \times \times \cdots]))\right)=R([\cdots \otimes \times \otimes \otimes \cdots])$,

(4) $Y_{i+2, i+3} Y_{i+1, i}\left(t_{c_{i}}^{-1}(R([\cdots \times \times \otimes \times \cdots]))\right)=R([\cdots \otimes \otimes \times \otimes \cdots])$,

(5) $Y_{i+1, i+2} Y_{i, i+2} Y_{i+3, i+2}^{-1} Y_{i+2, i+3}^{-1} Y_{i, i+3}^{-1}\left(t_{c_{i}}^{-1}(R([\cdots \otimes \times \otimes \times \cdots]))\right)$ $=R([\cdots \otimes \times \otimes \times \cdots])$,

(7) $Y_{i, i+3} Y_{i+1, i+3} Y_{i+2, i+3}\left(t_{c_{i}}^{-1}(R([\cdots \otimes \otimes \otimes \times \cdots]))\right)=R([\cdots \times \times \times \otimes \cdots])$,

(8) $Y_{i+3, i+2} Y_{i+1, i} Y_{i+2, i} Y_{i+2, i+1}^{-1}\left(t_{c_{i}}(R([\cdots \times \times \times \otimes \cdots]))\right)=R([\cdots \otimes \otimes \otimes \times \cdots])$,

(9) $Y_{i+2, i+3} Y_{i+1, i} Y_{i+3, i} Y_{i+3, i+1}^{-1} Y_{i+3, i+2} Y_{i+2, i+3} Y_{i+1, i+3} Y_{i+1, i+2}^{-1} Y_{i, i+3}$ $\cdot Y_{i, i+2}^{-1} Y_{i, i+1}\left(t_{c_{i}}^{-1}(R([\cdots \otimes \times \times \otimes \cdots]))\right)=R([\cdots \otimes \times \times \otimes \cdots])$,

(10) $Y_{i+2, i+1} Y_{i+3, i+1} Y_{i, i+3}^{-1} Y_{i+1, i}^{-1} Y_{i+3, i}^{-1}\left(t_{c_{i}}(R([\cdots \times \otimes \times \otimes \cdots]))\right)$ $=R([\cdots \times \otimes \times \otimes \cdots])$,

(11) $Y_{i, i+2} Y_{i+1, i+2} Y_{i+3, i+2} Y_{i+2, i} Y_{i+2, i+1}^{-1}\left(t_{c_{i}}(R([\cdots \otimes \otimes \times \otimes \cdots]))\right)$ $=R([\cdots \times \times \otimes \times \cdots])$,

(13) $Y_{i+3, i+1} Y_{i+2, i+1} Y_{i, i+1} Y_{i+1, i+3} Y_{i+1, i+2}^{-1}\left(t_{c_{i}}^{-1}(R([\cdots \otimes \times \otimes \otimes \cdots]))\right)$ $=R([\cdots \times \otimes \times \times \cdots])$,

(14) $Y_{i+3, i} Y_{i+2, i} Y_{i+1, i}\left(t_{c_{i}}(R([\cdots \times \otimes \otimes \otimes \cdots]))\right)=R([\cdots \otimes \times \times \times \cdots])$.

Proof of Lemma 4.4 Let $Y_{m, a}$ be a $Y$-homeomorphism whose leg is an r-circle. By Corollary 4.8, there is an element $\phi \in G_{g}$ such that $\phi(m)=R([\oplus-+\cdots])$ or $R([+\ominus+\cdots])$. Therefore $Y_{m, a}$ is $G_{g}$-equivalent to a short-leg $Y$-homeomorphism. By Lemma 4.9, we get our conclusion.

\subsection{Szepietowski's generators for $\Gamma_{2}\left(N_{g}\right)$}

We review the finite system of generators for $\Gamma_{2}\left(N_{g}\right)$ introduced in [14]. For each nonempty subset $I=\left\{i_{1}, i_{2}, \ldots, i_{k}\right\}$ of $\{1, \ldots, g\}$, let $\alpha_{I}$ be the simple closed curve shown in Figure 13. If $I=\{i\}$, we write $\alpha_{i}$ instead of $\alpha_{\{i\}}$.

Theorem 4.10 (Szepietowski [14, Theorem 3.2]) For $g \geq 4, \Gamma_{2}\left(N_{g}\right)$ is generated by the following elements.

(1) $Y_{\alpha_{i}, \alpha_{\{i, j\}}}$ for $i \neq j$,

(2) $Y_{\alpha_{\{i, j, k\}}, \alpha_{\{i, j, k, l\}}}$ for $i<j<k<l$.

The group $\Gamma_{2}\left(N_{3}\right)$ is generated by the elements in (1). 

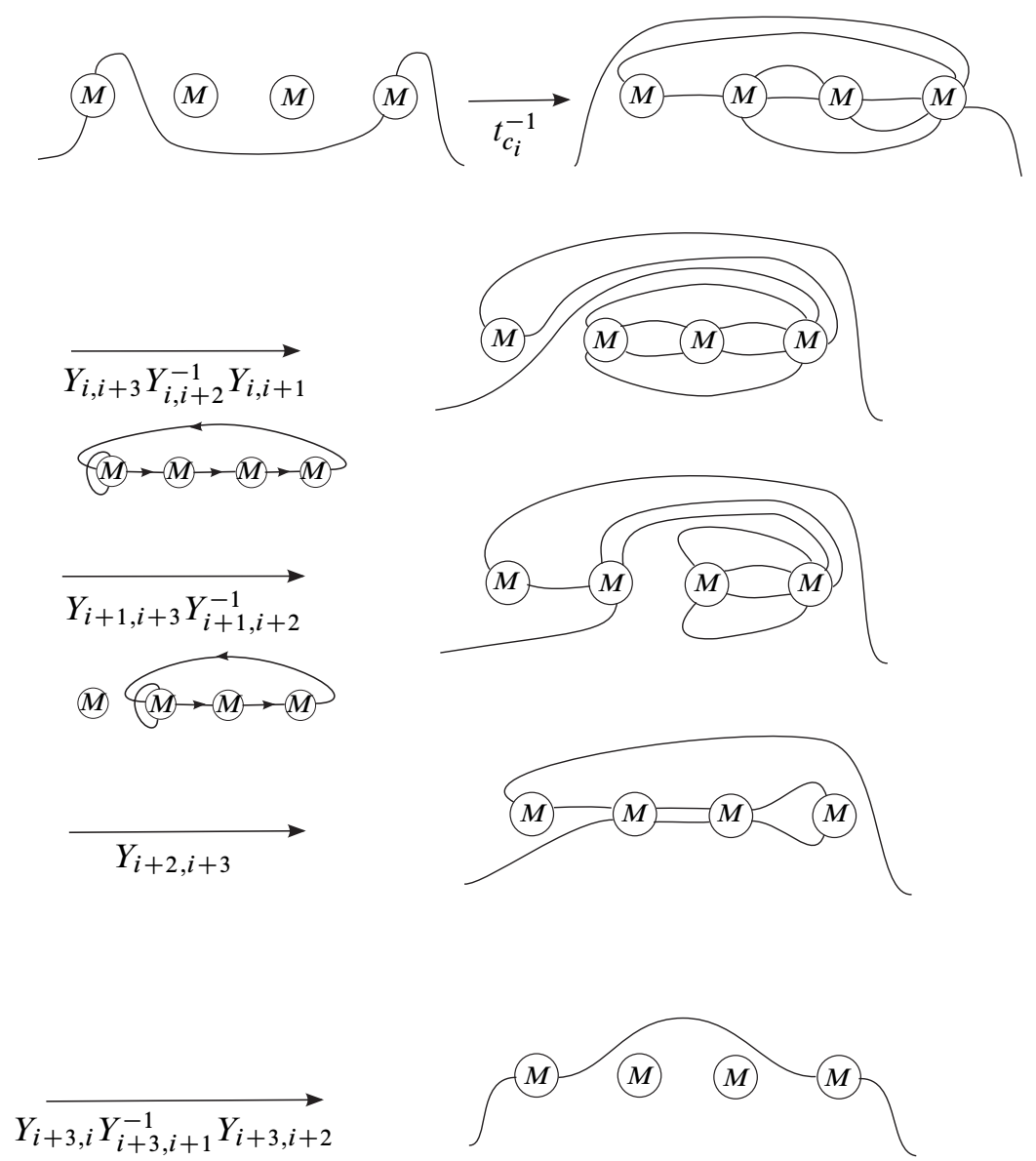

$$
(M-(M) \leftarrow M-(M)
$$

$$
\underset{Y_{i+2, i+3} Y_{i+1, i}}{\longrightarrow}
$$

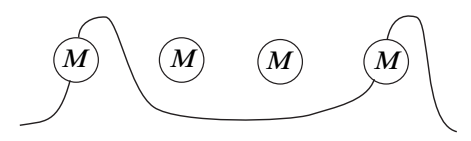

Figure 12

We show:

Lemma 4.11 For arbitrary $i<j<k<l, Y_{\alpha_{\{i, j, k\}}, \alpha_{\{i, j, k, l\}}}$ is $G_{g}$-equivalent to a short-leg $Y$-homeomorphism.

Proof It suffices to show that, for every $i<j<k, \alpha_{\{i, j, k\}}$ is $G_{g}$-equivalent to $\alpha_{1}$ or $\alpha_{2}$. By drawing figures, we see when $i>2, Y_{i, i-2}^{-1} Y_{i-1, i-2}^{-1} t_{d_{i-2}} \alpha_{\{i, j, k\}}=\alpha_{\{i-2, j, k\}}$ 


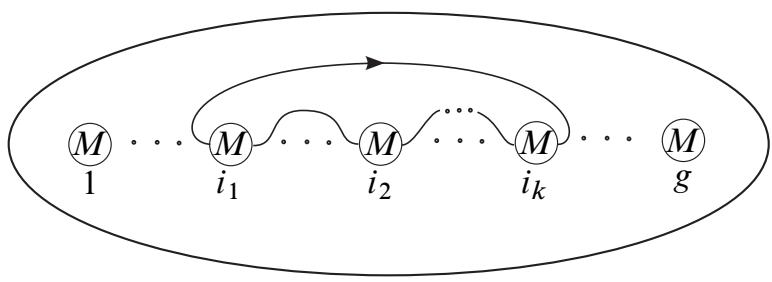

Figure 13. The curve $\alpha_{I}$ for $I=\left\{i_{1}, i_{2}, \ldots, i_{k}\right\}$

and when $i<j-2, Y_{j, j-2}^{-1} Y_{j-1, j-2}^{-1} t_{d_{j-2}} \alpha_{\{i, j, k\}}=\alpha_{\{i, j-2, k\}}$; when $j<k-2$, $Y_{k, k-2}^{-1} Y_{k-1, k-2}^{-1} t_{d_{k-2}} \alpha_{\{i, j, k\}}=\alpha_{\{i, j, k-2\}}$. By applying the above action of $G_{g}$ on $\alpha_{\{i, j, k\}}$, we see that $\alpha_{\{i, j, k\}}$ is $G_{g}$-equivalent to $\alpha_{\{1,3,4\}}, \alpha_{\{1,2,3\}}, \alpha_{\{2,3,5\}}, \alpha_{\{2,4,6\}}$, $\alpha_{\{1,3,5\}}, \alpha_{\{1,2,4\}}, \alpha_{\{2,3,4\}}$ or $\alpha_{\{2,4,5\}}$. By drawing figures of the action of $G_{g}$ on the above 8 circles, we can check that former 4 circles are $G_{g}$-equivalent to $\alpha_{1}$ and last 4 circles are $G_{g}$-equivalent to $\alpha_{2}$. For example, Figure 14 indicates that $\alpha_{\{1,3,5\}}$, $\alpha_{\{2,4,5\}}$ and $\alpha_{\{2,3,4\}}$ are $G_{g}$-equivalent to $\alpha_{2}$.
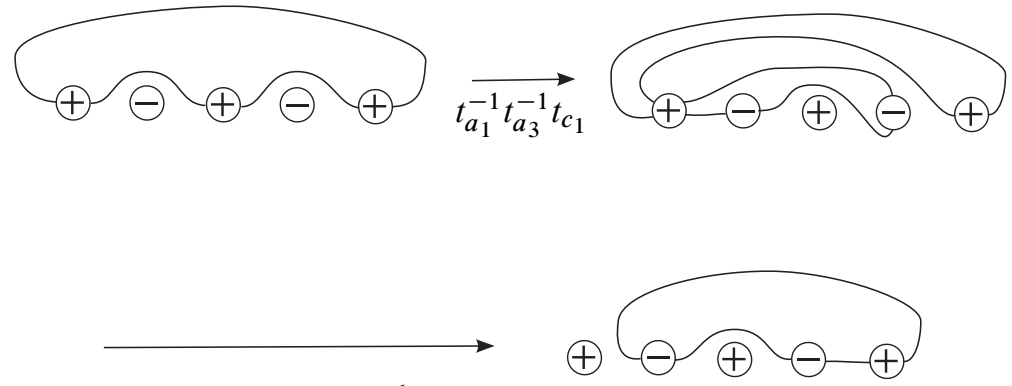

$Y$-homeo. about
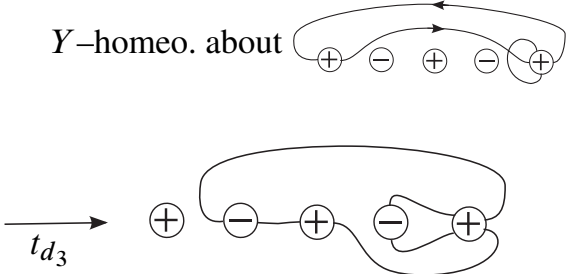

†
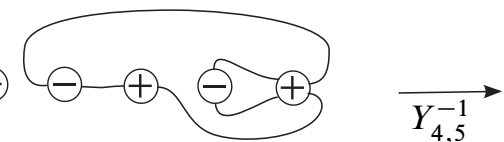

$\oplus$
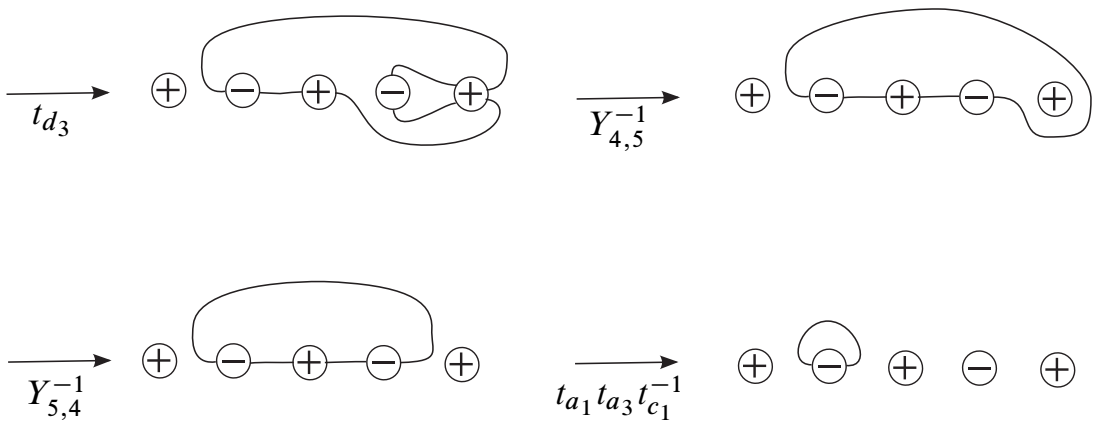

Figure 14. The first circle is $\alpha_{\{1,3,5\}}$, the third circle is $\alpha_{\{2,4,5\}}$, and the sixth circle is $\alpha_{\{2,3,4\}}$. 
Every $Y$-homeomorphism $Y$ is an element of $\Gamma_{2}\left(N_{g}\right)$, and by Theorem 4.10 and Lemma 4.11, we can express $Y$ as a product of $Y$-homeomorphisms which are $G_{g}$-equivalent to short-leg $Y$-homeomorphisms. Hence, Lemma 4.4 follows.

\subsection{Generators for $\mathcal{O}_{g}\left(q_{o s}\right)$}

For $a \in H_{1}\left(N_{g} ; \mathbb{Z}_{2}\right)$, we define the transvection $T_{a}: H_{1}\left(N_{g} ; \mathbb{Z}_{2}\right) \rightarrow H_{1}\left(N_{g} ; \mathbb{Z}_{2}\right)$ about $a$ by $T_{a}(x)=x+(x \cdot a)_{2} a$, where $(\cdot)_{2}$ means the mod-2 intersection form. We remark that if $l$ is an A-circle on $N_{g}$ such that $[l]=a \in H_{1}\left(N_{g} ; \mathbb{Z}_{2}\right)$, then $\left(t_{l}\right)_{*}=T_{a}$. If we find a system of generators $\left\{S_{1}, \ldots, S_{k}\right\}$ for $\mathcal{O}_{g}\left(q_{o s}\right)$ and elements $\sigma_{1}, \ldots, \sigma_{k}$ of $\mathcal{M}\left(N_{g}\right)$ such that $\left(\sigma_{i}\right)_{*}=S_{i}$ in $\operatorname{Aut}\left(H_{1}\left(N_{g} ; \mathbb{Z}_{2}\right)\right)$, then, by the short exact sequence (1) and Corollary 4.5, we see that $\mathcal{N}_{g}\left(q_{o s}\right)$ is generated by $G_{g} \cup\left\{\sigma_{1}, \ldots, \sigma_{k}\right\}$.

Theorem 4.12 (Nowik [12, Theorem 3.2]) $\mathcal{O}_{g}\left(q_{o s}\right)$ is generated by the set of elements of the following two forms:

(1) $T_{a}$ for $a \in H_{1}\left(N_{g} ; \mathbb{Z}_{2}\right)$ with $q_{o s}(a)=2$,

(2) $T_{a} T_{b} T_{a+b}$ for $a, b \in H_{1}\left(N_{g} ; \mathbb{Z}_{2}\right)$ with $q_{o s}(a)=q_{o s}(b)=q_{o s}(a+b)=0$.

Let $\left\{x_{1}, \ldots, x_{g}\right\}$ be the basis of $H_{1}\left(N_{g} ; \mathbb{Z}_{2}\right)$ which is introduced in Figure 1 . We obtain a finite system of generators for $\mathcal{O}_{g}\left(q_{o s}\right)$ explicitly.

Lemma $4.13 \mathcal{O}_{g}\left(q_{o s}\right)$ is generated by

$$
\begin{array}{ll}
T_{x_{i}+x_{i+2}}, & i=1, \ldots, g-2, \\
T_{x_{i}+x_{i+1}} T_{x_{i+2}+x_{i+3}} T_{x_{i}+x_{i+1}+x_{i+2}+x_{i+3}}, & i=1, \ldots, g-3 .
\end{array}
$$

Proof Write any element $v$ of $H_{1}\left(N_{g} ; \mathbb{Z}_{2}\right)$ as $v=x_{i_{1}}+\cdots+x_{i_{m}}$ such that $i_{1}<\cdots<i_{m}$ and call $m$ the length of $v$, or as $v=\left(x_{2 j_{1}+1}+\cdots+x_{2 j_{k}+1}\right) \oplus\left(x_{2 j_{k+1}}+\cdots+x_{2 j_{m}}\right)$ such that $j_{1}<\cdots<j_{k}, j_{k+1}<\cdots<j_{m}$ and call $\left(x_{2 j_{1}+1}+\cdots+x_{2 j_{k}+1}\right)$ the odd part of $v$, and $\left(x_{2 j_{k+1}}+\cdots+x_{2 j_{m}}\right)$ the even part of $v$. Two elements $v, w$ of $H_{1}\left(N_{g} ; \mathbb{Z}_{2}\right)$ are (4)-equivalent $v \sim{ }_{(4)} w$ if there is a product $T$ of (4) such that $T(v)=w$, and define (5)-equivalence $v \sim_{(5)} w$ and (4)-and (5)-equivalence $v \sim_{(4),(5)} w$ in the same way. We remark that if $v \sim_{(4),(5)} w$ then there is a product $T$ of (4) and (5) such that $T_{w}=T T_{v} T$.

Any element (4) acts only on the odd part of $v$ or only on the even part of $v$. For example, when $i<j<k$,

$$
\begin{aligned}
T_{x_{2 j-1}+x_{2 j+1}}\left(\left(\cdots+x_{2 i-1}+x_{2 j+1}\right.\right. & \left.\left.+x_{2 k+1}+\cdots\right) \oplus(\cdots)\right) \\
& =\left(\left(\cdots+x_{2 i-1}+x_{2 j-1}+x_{2 k+1}+\cdots\right) \oplus(\cdots)\right) .
\end{aligned}
$$


Therefore, if we define $l_{o}(v)$ to be the length of the odd part of $v$, and $l_{e}(v)$ to

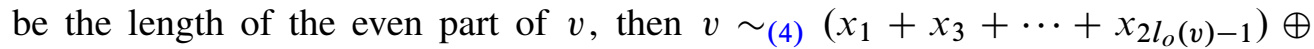
$\left(x_{2}+x_{4}+\cdots+x_{2 l_{e}(v)}\right)$. Hence, $v \sim_{(4)} w$ if and only if $l_{o}(v)=l_{o}(w)$ and $l_{e}(v)=l_{e}(w)$.

When $p<i, i+3<s,\{i, i+1\}=\left\{q, q^{\prime}\right\}$ and $\{i+2, i+3\}=\left\{r, r^{\prime}\right\}$, the element (5) acts as follows.

$$
\begin{aligned}
T_{x_{i}+x_{i+1}} T_{x_{i+2}+x_{i+3}} & T_{x_{i}+x_{i+1}+x_{i+2}+x_{i+3}}\left(\cdots+x_{p}+x_{q}+x_{r}+x_{s}+\cdots\right) \\
& =T_{x_{i}+x_{i+1}} T_{x_{i+2}+x_{i+3}}\left(\cdots+x_{p}+x_{q}+x_{r}+x_{s}+\cdots\right) \\
& =\cdots+x_{p}+x_{q^{\prime}}+x_{r^{\prime}}+x_{s}+\cdots, \\
T_{x_{i}+x_{i+1}} T_{x_{i+2}+x_{i+3}} & T_{x_{i}+x_{i+1}+x_{i+2}+x_{i+3}}\left(\cdots+x_{p}+x_{q}+x_{s}+\cdots\right) \\
& =T_{x_{i}+x_{i+1}} T_{x_{i+2}+x_{i+3}}\left(\cdots+x_{p}+x_{q^{\prime}}+x_{r}+x_{r^{\prime}}+x_{s}+\cdots\right) \\
& =\cdots+x_{p}+x_{q}+x_{r}+x_{r^{\prime}}+x_{s}+\cdots, \\
T_{x_{i}+x_{i+1}} T_{x_{i+2}+x_{i+3}} & T_{x_{i}+x_{i+1}+x_{i+2}+x_{i+3}}\left(\cdots+x_{p}+x_{r}+x_{s}+\cdots\right) \\
& =T_{x_{i}+x_{i+1}} T_{x_{i+2}+x_{i+3}}\left(\cdots+x_{p}+x_{q}+x_{q^{\prime}}+x_{r^{\prime}}+x_{s}+\cdots\right) \\
& =\cdots+x_{p}+x_{q}+x_{q^{\prime}}+x_{r}+x_{s}+\cdots .
\end{aligned}
$$

We will show that every element of the first form in Theorem 4.12 is a product of (4) and (5). Let $a$ be an element of $H_{1}\left(N_{g} ; \mathbb{Z}_{2}\right)$ such that $q_{o s}(a)=2$. Then, $2 \equiv l_{o}(a)-l_{e}(a) \bmod 4$. Therefore, there are two cases $l_{o}(a)=l_{e}(a)+4 t+2$ or $l_{e}(a)=l_{o}(a)+4 t+2(t \in \mathbb{Z})$. For the first case,

$a \sim_{(4)}\left(x_{1}+x_{3}\right)+\left(x_{4}+x_{5}\right)+\cdots+\left(x_{2 i}+x_{2 i+1}\right)+\left(x_{l}+x_{l+2}+x_{l+4}+x_{l+6}\right)$

$$
+\cdots+\left(x_{m}+x_{m+2}+x_{m+4}+x_{m+6}\right) \text {. }
$$

For the second case,

$\begin{aligned} a \sim_{(4)}\left(x_{2}+x_{4}\right)+\left(x_{5}+x_{6}\right)+\cdots+\left(x_{2 i+1}\right. & \left.+x_{2 i+2}\right)+\left(x_{l}+x_{l+2}+x_{l+4}+x_{l+6}\right) \\ & +\cdots+\left(x_{m}+x_{m+2}+x_{m+4}+x_{m+6}\right) .\end{aligned}$

We see

$$
\begin{array}{r}
\left(x_{2}+x_{4}\right)+\left(x_{5}+x_{6}\right)+\cdots+\left(x_{2 i+1}+x_{2 i+2}\right)+\left(x_{l}+x_{l+2}+x_{l+4}+x_{l+6}\right) \\
+\cdots+\left(x_{m}+x_{m+2}+x_{m+4}+x_{m+6}\right) \\
\sim_{(5)}\left(x_{1}+x_{3}\right)+\left(x_{5}+x_{6}\right)+\cdots+\left(x_{2 i+1}+x_{2 i+2}\right)+\left(x_{l}+x_{l+2}+x_{l+4}+x_{l+6}\right) \\
+\cdots+\left(x_{m}+x_{m+2}+x_{m+4}+x_{m+6}\right) \\
\sim_{(4)}\left(x_{1}+x_{3}\right)+\left(x_{4}+x_{5}\right)+\cdots+\left(x_{2 i}+x_{2 i+1}\right)+\left(x_{l}+x_{l+2}+x_{l+4}+x_{l+6}\right) \\
+\cdots+\left(x_{m}+x_{m+2}+x_{m+4}+x_{m+6}\right)
\end{array}
$$


where $\sim_{(5)}$ is by $T_{x_{1}+x_{2}} T_{x_{3}+x_{4}} T_{x_{1}+x_{2}+x_{3}+x_{4}}$. Therefore, it suffices to consider the first case. We see

$$
\begin{aligned}
\left(x_{l}+x_{l+2}+x_{l+4}+x_{l+6}\right) \sim(5)\left(x_{l}+x_{l+2}+x_{l+3}+x_{l+5}\right) & \sim(5)\left(x_{l}+x_{l+5}\right) \\
& \sim_{(4)}\left(x_{l}+x_{l+1}\right),
\end{aligned}
$$

where the first $\sim_{(5)}$ is by $T_{x_{l+3}+x_{l+4}} T_{x_{l+5}+x_{l+6}} T_{x_{l+3}+x_{l+4}+x_{l+5}+x_{l+6}}$, and the second $\sim_{(5)}$ is by $T_{x_{l+2}+x_{l+3}} T_{x_{l+4}+x_{l+5}} T_{x_{l+2}+x_{l+3}+x_{l+4}+x_{l+5}}$. Hence, $a \sim_{(4),(5)}$ $\left(x_{1}+x_{3}\right)+\left(x_{4}+x_{5}\right)+\cdots+\left(x_{2 n}+x_{2 n+1}\right)$. Furthermore,

$$
\begin{aligned}
\left(x_{1}+x_{3}\right)+\left(x_{4}+x_{5}\right) & +\left(x_{6}+x_{7}\right)+\cdots+\left(x_{2 n}+x_{2 n+1}\right) \\
& =\left(x_{1}+x_{3}+x_{4}\right)+x_{5}+\left(x_{6}+x_{7}\right)+\cdots+\left(x_{2 n}+x_{2 n+1}\right) \\
& \sim(5) x_{1}+x_{5}+\left(x_{6}+x_{7}\right)+\cdots+\left(x_{2 n}+x_{2 n+1}\right) \\
& \sim(4) x_{1}+x_{3}+\left(x_{4}+x_{5}\right)+\cdots+\left(x_{2(n-1)}+x_{2(n-1)+1}\right),
\end{aligned}
$$

where $\sim_{(5)}$ is by $T_{x_{1}+x_{2}} T_{x_{3}+x_{4}} T_{x_{1}+x_{2}+x_{3}+x_{4}}$. Therefore, by the induction on $n$, we see $a \sim_{(4),(5)} x_{1}+x_{3}$. Hence $T_{a}$ is a product of (4) and (5) if $q_{o s}(a)=2$.

We will show that every element of the second form in Theorem 4.12 is a product of (4) and (5). Let $a$ and $b$ be elements of $H_{1}\left(N_{g} ; \mathbb{Z}_{2}\right)$ such that $q_{o s}(a)=q_{o s}(b)=$ $q_{o s}(a+b)=0$. Then $0=q_{o s}(a+b)=q_{o s}(a)+q_{o s}(b)+(a \cdot b)_{2}=(a \cdot b)_{2}$, $0=q_{o s}(2 a)=q_{o s}(a)+q_{o s}(a)+(a \cdot a)_{2}=(a \cdot a)_{2}$, by the same reason, $0=(b \cdot b)_{2}$, hence $(a+b \cdot a)_{2}=(a+b \cdot b)_{2}=0$. Therefore, $T_{a}, T_{b}$ and $T_{a+b}$ commute each other. For the pairs $\left[a_{1}, b_{1}\right]$ and $\left[a_{2}, b_{2}\right]$ of elements of $H_{1}\left(N_{g} ; \mathbb{Z}_{2}\right)$ which satisfies $q_{o s}\left(a_{i}\right)=q_{o s}\left(b_{i}\right)=q_{o s}\left(a_{i}+b_{i}\right)=0(i=1,2)$, we define the equivalence $\left[a_{1}, b_{1}\right] \sim_{(4)}\left[a_{2}, b_{2}\right]$ if there is a product $T$ of (4) such that $T\left(a_{1}\right)=a_{2}$ and $T\left(b_{1}\right)=b_{2}$. The equivalences $\left[a_{1}, b_{1}\right] \sim_{(5)}\left[a_{2}, b_{2}\right]$ and $\left[a_{1}, b_{1}\right] \sim_{(4),(5)}\left[a_{2}, b_{2}\right]$ are defined in the same way.

Let $a, b$ be elements of $H_{1}\left(N_{g} ; \mathbb{Z}_{2}\right)$ such that $q_{o s}(a)=q_{o s}(b)=q_{o s}(a+b)=0$. By the same argument applied for elements of the first from in Theorem 4.12, we see $a \sim_{(4),(5)}\left(x_{1}+x_{2}\right)+\cdots+\left(x_{2 n-1}+x_{2 n}\right)$.

If $2 n \neq g$, then

$$
\begin{aligned}
\left(x_{1}+x_{2}\right)+\cdots+\left(x_{2 n-3}\right. & \left.+x_{2 n-2}\right)+\left(x_{2 n-1}+x_{2 n}\right) \\
& =\left(x_{1}+x_{2}\right)+\cdots+x_{2 n-3}+\left(x_{2 n-2}+x_{2 n-1}+x_{2 n}\right) \\
& \sim_{(5)}\left(x_{1}+x_{2}\right)+\cdots+x_{2 n-3}+x_{2 n} \\
& \sim_{(4)}\left(x_{1}+x_{2}\right)+\cdots+x_{2 n-3}+x_{2 n-2} \\
& =\left(x_{1}+x_{2}\right)+\cdots+\left(x_{2(n-1)-1}+x_{2(n-1)}\right),
\end{aligned}
$$

where $\sim_{(5)}$ is by $T_{x_{2 n-2}+x_{2 n-1}} T_{x_{2 n}+x_{2 n+1}} T_{x_{2 n-2}+x_{2 n-1}+x_{2 n}+x_{2 n+1}}$. Therefore, by the induction on $n$, we see $a \sim_{(4),(5)} x_{1}+x_{2}$. Hence, $[a, b] \sim_{(4),(5)}\left[x_{1}+x_{2}, b^{\prime}\right]$. 
Since $0=\left(x_{1}+x_{2} \cdot b^{\prime}\right)_{2}, b^{\prime}=x_{1}+x_{2}+x_{l}+\cdots+x_{m}$ or $b^{\prime}=x_{l}+\cdots+x_{m}$ where $l \geq 3$. For these two cases, $T_{x_{1}+x_{2}} T_{b^{\prime}} T_{x_{1}+x_{2}+b^{\prime}}=T_{x_{1}+x_{2}} T_{x_{1}+x_{2}+b^{\prime}} T_{b^{\prime}}$ are the same. Therefore, we may suppose $b^{\prime}=x_{l}+\cdots+x_{m}$. We see $[a, b] \sim_{(4),(5)}$ $\left[x_{1}+x_{2},\left(x_{3}+x_{4}\right)+\cdots+\left(x_{2 k-1}+x_{2 k}\right)\right]$, by applying (4) and (5) whose transvections are about $v$ such that $\operatorname{supp}(v)$ contains neither $x_{1}$ nor $x_{2}$. If $2 k \neq g$, by applying to $b$ the same argument as to $a$, we see $[a, b] \sim_{(4),(5)}\left[x_{1}+x_{2}, x_{3}+x_{4}\right]$. If $2 k=g$, then $T_{a} T_{b} T_{a+b}$ is equal to $T_{x_{1}+x_{2}} T_{x_{3}+\cdots+x_{g}} T_{x_{1}+x_{2}+x_{3}+\cdots+x_{g}}$. In the last paragraph of this proof, we show that $T_{x_{1}+x_{2}} T_{x_{3}+\cdots+x_{g}} T_{x_{1}+x_{2}+x_{3}+\cdots+x_{g}}$ is a product of (4) and (5).

If $2 n=g$, then $\left(v \cdot\left(x_{1}+x_{2}\right)+\cdots+\left(x_{2 n-1}+x_{2 n}\right)\right)_{2}=0$ for every $v \in H_{1}\left(N_{g} ; \mathbb{Z}_{2}\right)$ used for the transvections in (4) and (5). Therefore, we see

$[a, b] \sim(4),(5)\left[\left(x_{1}+x_{2}\right)+\cdots+\left(x_{g-1}+x_{g}\right),\left(x_{2 i+1}+x_{2 i+2}\right)+\cdots+\left(x_{g-1}+x_{g}\right)\right]$.

This means that $T_{a} T_{b} T_{a+b}=T_{a+b} T_{b} T_{a}$ is conjugate to

$$
T_{\left(x_{1}+x_{2}\right)+\cdots+\left(x_{2 i-1}+x_{2 i}\right)} T_{\left(x_{2 i+1}+x_{2 i+2}\right)+\cdots+\left(x_{g-1}+x_{g}\right)} T_{\left(x_{1}+x_{2}\right)+\cdots+\left(x_{g-1}+x_{g}\right)}
$$

by (4) and (5). Moreover,

$\left[\left(\left(x_{1}+x_{2}\right)+\cdots+\left(x_{2 i-3}+x_{2 i-2}\right)+\left(x_{2 i-1}+x_{2 i}\right)\right)\right.$,

$$
\left.\left(x_{2 i+1}+x_{2 i+2}\right)+\cdots+\left(x_{g-1}+x_{g}\right)\right]
$$

$\sim_{(5)}\left[\left(x_{1}+x_{2}\right)+\cdots+x_{2 i-3}+x_{2 i}\right.$,

$$
\left.x_{2 i-2}+x_{2 i-1}+\left(x_{2 i+1}+x_{2 i+2}\right)+\cdots+\left(x_{g-1}+x_{g}\right)\right]
$$

$\sim_{(4)}\left[\left(x_{1}+x_{2}\right)+\cdots+\left(x_{2 i-3}+x_{2 i-2}\right)\right.$,

$$
\left.\left(x_{2 i-1}+x_{2 i}\right)+\left(x_{2 i+1}+x_{2 i+2}\right)+\cdots+\left(x_{g-1}+x_{g}\right)\right] \text {, }
$$

where $\sim_{(5)}$ is by $T_{x_{2 i-2}+x_{2 i-1}} T_{x_{2 i}+x_{2 i+1}} T_{x_{2 i-2}+x_{2 i-1}+x_{2 i}+x_{2 i+1}}$, and $\sim_{(4)}$ is by $T_{x_{2 i-2}+x_{2 i}}$. By repeatedly applying the above argument, we see that $T_{a} T_{b} T_{a+b}$ is conjugate to $T_{x_{1}+x_{2}} T_{x_{3}+\cdots+x_{g}} T_{x_{1}+x_{2}+x_{3}+\cdots+x_{g}}$ by (4) and (5).

When $g$ is even and $g \geq 6$, by checking the action of transvections on the basis $\left\{x_{1}, \ldots, x_{g}\right\}$ of $H_{1}\left(N_{g} ; \mathbb{Z}_{2}\right)$, we show $T_{x_{1}+x_{2}} T_{x_{3}+\cdots+x_{g}} T_{x_{1}+x_{2}+x_{3}+\cdots+x_{g}}=$ $T_{x_{1}+x_{2}} T_{x_{3}+x_{4}} T_{x_{1}+x_{2}+x_{3}+x_{4}} \cdot T_{x_{1}+x_{2}} T_{x_{5}+\cdots+x_{g}} T_{x_{1}+x_{2}+x_{5}+\cdots+x_{g}}$. Since we have $\left[x_{1}+x_{2}, x_{5}+\cdots+x_{g}\right] \sim_{(4)}\left[x_{1}+x_{2}, x_{3}+\cdots+x_{g-2}\right]$, we see that the element $T_{x_{1}+x_{2}} T_{x_{5}+\cdots+x_{g}} T_{x_{1}+x_{2}+x_{5}+\cdots+x_{g}}$ is a product of (4) and (5) by using the argument for the case where $2 k(=g-2) \neq g$.

Since $\left(t_{d_{i}}\right)_{*}=T_{x_{i}+x_{i+2}},\left(t_{a_{i}} t_{a_{i+2}} t_{c_{i}}\right)_{*}=T_{x_{i}+x_{i+1}} T_{x_{i+2}+x_{i+3}} T_{x_{i}+x_{i+1}+x_{i+2}+x_{i+3}}$, and $t_{d_{i}}, t_{a_{i}} t_{a_{i+2}} t_{c_{i}} \in G_{g}$, we see $G_{g}=\mathcal{N}_{g}\left(q_{o s}\right)$, hence Theorem 4.1 follows. 
Acknowledgements The author wishes to express his gratitude to Professors Akio Kawauchi and Masamichi Takase for advising the author for considering on $o$-standard embeddings and Professor Błażej Szepietowski for informing and sending the author his preprint [14]. The author would also like to thank the referee, whose comments and corrections improved the paper. This research was supported by Grant-in-Aid for Scientific Research (C) number 20540083, Japan Society for the Promotion of Science.

\section{References}

[1] J S Birman, Mapping class groups and their relationship to braid groups, Comm. Pure Appl. Math. 22 (1969) 213-238 MR0243519

[2] D R J Chillingworth, A finite set of generators for the homeotopy group of a nonorientable surface, Proc. Cambridge Philos. Soc. 65 (1969) 409-430 MR0235583

[3] L Guillou, A Marin, Une extension d'un théorème de Rohlin sur la signature, C. R. Acad. Sci. Paris Sér. A 285 (1977) A95-A98 MR0440566

[4] S Hirose, On diffeomorphisms over surfaces trivially embedded in the 4-sphere, Algebr. Geom. Topol. 2 (2002) 791-824 MR1928177

[5] S Hirose, Surfaces in the complex projective plane and their mapping class groups, Algebr. Geom. Topol. 5 (2005) 577-613 MR2153115

[6] S Hirose, A Yasuhara, Surfaces in 4-manifolds and their mapping class groups, Topology 47 (2008) 41-50 MR2415773

[7] D Johnson, The structure of the Torelli group I: A finite set of generators for $\mathscr{I}$, Ann. of Math. 118 (1983) 423-442 MR727699

[8] W B R Lickorish, Homeomorphisms of non-orientable two-manifolds, Proc. Cambridge Philos. Soc. 59 (1963) 307-317 MR0145498

[9] W B R Lickorish, On the homeomorphisms of a non-orientable surface, Proc. Cambridge Philos. Soc. 61 (1965) 61-64 MR0169221

[10] Y Matsumoto, An elementary proof of Rochlin's signature theorem and its extension by Guillou and Marin, from: "À la recherche de la topologie perdue", (L Guillou, A Marin, editors), Progr. Math. 62, Birkhäuser, Boston, MA (1986) 119-139 MR900248

[11] J M Montesinos, On twins in the four-sphere I, Quart. J. Math. Oxford Ser. 34 (1983) 171-199 MR698205

[12] T Nowik, Immersions of non-orientable surfaces, Topology Appl. 154 (2007) 18811893 MR2319260

[13] B Szepietowski, Crosscap slides and the level 2 mapping class group of a nonorientable surface arXiv:1006.5410 
[14] B Szepietowski, A finite generating set for the level 2 mapping class group of a nonorientable surface arXiv:1108.3927

Department of Mathematics, Faculty of Science and Technology, Tokyo University of Science Noda, Chiba 278-8510, Japan

hirose_susumu@ma.noda.tus.ac.jp

Received: 11 September $2011 \quad$ Revised: 24 October 2011 